\title{
Endothelin-B Receptors and Left Ventricular Dysfunction after Regional versus Global Ischaemia-Reperfusion in Rat Hearts
}

\author{
Sofia-Iris Bibli, ${ }^{1,2}$ Eleni V. Toli, ${ }^{2,3}$ Agapi D. Vilaeti, ${ }^{2,3}$ Varnavas C. Varnavas, ${ }^{2,4}$ Giannis G. \\ Baltogiannis, ${ }^{2,3}$ Apostolos Papalois, ${ }^{2,5}$ Zenon S. Kyriakides, ${ }^{2,6}$ and Theofilos M. Kolettis ${ }^{2,3}$ \\ ${ }^{1}$ Department of Pharmaceutical Chemistry, University of Athens School of Pharmacy, University Campus, \\ Zografou, Athens 15771, Greece \\ ${ }^{2}$ Cardiovascular Research Institute, Zoodoxos, Ioannina 45500, Greece \\ ${ }^{3}$ Department of Cardiology, University of Ioannina, 1 Stavrou Niarxou Avenue, Ioannina 45110, Greece \\ ${ }^{4}$ Department of Cardiology, University Hospital, 55 Hufelandstrasse, Essen 45122, Germany \\ ${ }^{5}$ Experimental Research Center ELPEN, 95 Marathonos Avanue, Pikermi, Athens 19009, Greece \\ ${ }^{6}$ Department of Cardiology, Athens Red Cross Hospital, 1 Erythrou Stavrou Street, Athens 11526, Greece
}

Correspondence should be addressed to Theofilos M. Kolettis, thkolet@cc.uoi.gr

Received 1 May 2012; Revised 23 May 2012; Accepted 1 June 2012

Academic Editor: Yasuo Matsumura

Copyright () 2012 Sofia-Iris Bibli et al. This is an open access article distributed under the Creative Commons Attribution License, which permits unrestricted use, distribution, and reproduction in any medium, provided the original work is properly cited.

\begin{abstract}
Background. Endothelin-1 (ET-1) is implicated in left ventricular dysfunction after ischaemia-reperfusion. ETA and ETB receptors mediate diverse actions, but it is unknown whether these actions depend on ischaemia type and duration. We investigated the role of ETB receptors after four ischaemia-reperfusion protocols in isolated rat hearts. Methods. Left ventricular haemodynamic variables were measured in the Langendorff-perfused model after 40- and 20-minute regional or global ischaemia, followed by 30 -minute reperfusion. Wild-type $(n=39)$ and ETB-deficient $(n=41)$ rats were compared. Infarct size was measured using fluorescent microspheres after regional ischaemia-reperfusion. Results. Left ventricular dysfunction was more prominent in ETBdeficient rats, particularly after regional ischaemia. Infarct size was smaller $(P=0.006)$ in wild-type $(31.5 \pm 4.4 \%)$ than ETBdeficient $(45.0 \pm 7.3 \%)$ rats after 40 minutes of regional ischaemia-reperfusion. Although the recovery of left ventricular function was poorer after 40-minute ischaemia-reperfusion, end-diastolic pressure in ETB-deficient rats was higher after 20 than after 40 minutes of regional ischaemia-reperfusion. Conclusion. ETB receptors exert cytoprotective effects in the rat heart, mainly after regional ischaemia-reperfusion. Longer periods of ischaemia suppress the recovery of left ventricular function after reperfusion, but the role of ETB receptors may be more important during the early phases.
\end{abstract}

\section{Introduction}

Myocardial infarction is the most common clinical manifestation of cardiovascular disease, which remains a leading cause of death worldwide [1]. Prompt restoration of coronary blood flow in the acute phase decreases acute and longterm morbidity and mortality. However, the major limitation of this strategy is reperfusion injury, defined as a second peak of myocardial necrosis, initiated after the onset of reperfusion [2].

Endothelin-1 (ET-1) is a 21-amino-acid peptide, first identified by Yanagisawa and coworkers in 1988 [3]; in addition to its potent vasoconstrictor properties, ET-1 acts also on ventricular myocytes [4]. The effects of ET-1 are mediated via two G-protein-coupled receptors, namely, endothelin-A (ETA) and endothelin-B (ETB), which are abundantly present in the myocardium [5]. ET-1 exerts most of its effects via ETA receptors, whereas the role of ETB receptors in various disease states is incompletely understood [4].

Previous reports have demonstrated elevated circulating ET-1 levels [6-8], as well as increased ET-1 expression in the infarct zone [9], in response to prolonged myocardial ischaemia. ET-1 increases intracellular calcium concentration in ventricular myocytes [10], a well-described factor resulting in cellular necrosis [11]. These effects, along with 
microvascular obstruction, implicate ET-1 as a mediator of reperfusion injury [12].

Based on the pathophysiologic role of ET-1 during ischaemia-reperfusion, a large number of experimental studies (reviewed in references [12-14]) have investigated the therapeutic potential of ET-receptor blockade in decreasing myocardial necrosis and reperfusion injury and improving left ventricular (LV) dysfunction. However, these studies yielded mixed results, ranging from neutral to remarkably beneficial effects. For example, Dagassan et al. [15] using the dual (ETA and ETB) receptor antagonist bosentan reported no changes in postischaemic LV haemodynamic variables, whereas Lee et al. [16] found a $44 \%$ reduction in infarct size after selective ETA receptor blockade. In addition to the variability in ischaemia-reperfusion protocols utilized, the most likely explanation for these discrepant findings is the diversity of agents examined, displaying different degrees of selectivity against ETA and ETB receptors.

Under normal conditions, ETB receptors mediate the release of vasodilators and contribute to ET-1 clearance from the circulation [17]. However, it remains uncertain whether these beneficial actions are maintained under ischaemic conditions. In 2005, an important study by Yamamoto et al. [18] examined the role of ETB receptors in ischaemiareperfusion, using the isolated, Langendorff-perfused ratheart model; they [18] concluded that ETB receptors are beneficial in this setting and ameliorate postischaemic LV dysfunction. However, two methodological issues should be considered in this work. First, prolonged ischaemic periods in the Langendorff-perfused rat-heart model result in pronounced and sustained LV dysfunction [19-22], raising some limitations in the study of haemodynamic responses. Moreover, the 40 -minute ischaemic period in the Langendorff-perfused model likely corresponds to prolonged ischaemia in the clinical scenario; however, such cases are observed progressively less frequently, given the refinement of emergency medical systems and the widespread use of thrombolysis and/or primary percutaneous coronary interventions [23]. Second and foremost, although extensively utilized, the model of zero-flow global myocardial ischaemia presents clear-cut disadvantages with respect to its relevance with acute coronary artery occlusion in patients [20-22].

The purpose of the present study was to further examine the pathophysiologic role of ETB receptors (a) on the infarct size and (b) on LV function after ischaemia-reperfusion. We used the isolated, Langendorff-perfused working heart model, which enables accurate assessment of LV systolic and diastolic function indices. This model is pertinent, as ET-1release has been demonstrated after ischaemia-reperfusion [24]. As in the study by Yamamoto et al. [18], we used a rat strain, carrying a naturally occurring deletion in the ETB receptor gene that abrogates the expression of functional ETB receptors.

In the present work, we hypothesized that the effects of ETB receptor activation may vary, depending on the type and duration of ischaemia. For this purpose, we examined LV haemodynamic variables after four ischaemia protocols, namely, after 40- and 20-minute regional or global ischaemia, all followed by 30 minutes of reperfusion.
Based on the considerations stated above, the selection of the 40- and 20-minute ischaemia intervals was based on their potential correspondence with delayed or timely (resp.) admission of patients after acute myocardial infarction, whereas the 30-minute-reperfusion interval is widely used in experimental models and enables comprehensive assessment of postischaemic LV dysfunction [20-22].

\section{Materials and Methods}

2.1. Animal Cohort. The total animal cohort comprised 80 male rats (20-24 weeks of age); previous experience from our laboratory [25-27] indicates that this age range provides favourable experimental conditions, not only in terms of perioperative mortality, but also in terms of heart dimensions. All animals received humane care, according to European legislation (European Union directive for the protection of animals used for scientific purposes, 2010/63/EU); they were housed 1-2 per cage, under optimal laboratory conditions (controlled temperature, humidity, and 12:12 hour-light:dark cycles), with free access to water and standard rodent chow.

Of the experimental animal cohort, 39 (20-23 weeks of age, weighing $240 \pm 2 \mathrm{~g}$ ) were wild type and 41 (2024 weeks of age, weighing $259 \pm 3 \mathrm{~g}$ ) were homozygous ETB deficient. The latter rat model has been characterized previously $[28,29]$, and a colony is maintained in our animal facilities, kindly provided by Professor M. Yanagisawa (University of Texas Southwestern Medical Center, Dallas, TX, USA). This rat strain carries a 301-bp deletion in ETB receptors, resulting in abnormal mRNA transcript that completely abrogates functional ETB receptor expression [30]. The absence of ETB receptors in this rat strain has been confirmed by polymerase chain reaction, as well as by in situ hybridization for ETB mRNA [29]. To prevent premature death of intestinal obstruction in these animals, dopamine $\beta$ hydroxylase promoter has been used to direct ETB transgene expression and to support normal enteric nervous system development [28]. With this intervention, the animals live into adulthood, but are deficient of ETB receptors in the cardiovascular system, making this rat strain a useful tool in the study of the pathophysiology of ET-1.

Further characterization of this ETB-deficient rat strain demonstrated markedly increased levels of immunoreactive circulating plasma ET-1 concentrations [29]. Additionally, higher ETA receptor protein expression was shown in homozygous, compared to heterozygous animals [31]; this difference was observed in vascular membrane from small mesenteric arteries, after western blot analysis [31]. Nonetheless, functional uncoupling has been observed between ETA receptor expression and activity [31], which may depend on the vascular bed examined [31,32]. As noted above, dopamine $\beta$-hydroxylase promoter control is absent in the cardiovascular system of this rat strain [28, 29]; thus, this "partially rescued" phenotype permits accurate evaluation of ETB receptors in experimental models of myocardial ischaemia. 
2.2. Isolated Rat-Heart Preparation. The animals were anaesthetized with an intramuscular injection of ketamine $(50 \mathrm{mg} / \mathrm{kg})$ and xylazine $(5 \mathrm{mg} / \mathrm{kg})$ and were euthanized by cervical dislocation. The low dosage of this anaesthetic protocol, coupled with its brief duration associated with the ex vivo experimental conditions, precludes significant effects on cardiac function. After a left lateral thoracotomy, the hearts were rapidly excised and were mounted on a Langendorff apparatus (ML870B2 system, ADInstruments, Oxfordshire, UK), as previously described [27, 33]. The preparations were perfused at a constant flow of $12.5 \mathrm{ml} / \mathrm{min}$ with Krebs-Henseleit solution at the following composition (in mmol/L): $\mathrm{NaCl}: 118.1, \mathrm{KCl}: 4.6, \mathrm{CaCl}_{2}: 2.5, \mathrm{MgSO}_{4}$ : 1.2, $\mathrm{KH}_{2} \mathrm{PO}_{4}: 1.2, \mathrm{NaHCO}_{3}: 24.8$, and glucose: 10.33 . The perfusate was bubbled continuously with a gas mixture of $95 \% \mathrm{O}_{2}$ and $5 \% \mathrm{CO}_{2}$. The $\mathrm{pH}$ and temperature were kept stable at 7.4 and $37^{\circ} \mathrm{C}$, respectively. To ensure comparable measurements, atrial pacing was performed at a rate of 300 beats per minute during the stabilization period and during reperfusion; as in previous reports, pacing was continued during regional [34], but not during global [27, 33] ischaemia.

2.3. Haemodynamic Measurements. A water-filled balloon, connected to a pressure transducer, was placed into the LV through a left atrial incision. The balloon volume was adjusted to achieve a stable LV end-diastolic pressure (EDP) of $10 \mathrm{mmHg}$. LV pressure signal was continuously recorded and analyzed online by a dedicated software program (Chart 5, version 5.4.2., ADInstruments, Oxfordshire, UK). After a stabilization period of 20 minutes, LV developed pressure (DP), defined as LV systolic minus diastolic pressure, EDP, and maximum positive (maximum $+d p / d t$ ) and negative (maximum $-d p / d t$ ) values of the first derivative of pressure were recorded for 30 minutes at 10-minute intervals, as previously published [18].

2.4. Experimental Protocol. Two series of experiments were performed, namely, global and regional ischaemia. In the first series, both wild-type and ETB-deficient rats underwent either 40 or 20 minutes of global ischaemia, followed by 30 minutes of reperfusion. Zero-flow global ischaemia was attained with turning off the aortic cannula and reperfusion with the restoration of flow. In the second series, both rat strains underwent either 40 or 20 minutes of regional ischaemia, followed by 30-minutes of reperfusion. Regional ischaemia was induced by left coronary artery ligation, as described, [35, 36]. Briefly, the left coronary artery was encircled with a 4-0 suture near its origin, extending from the pulmonary cone to the myocardium under the left atrial appendage; following these anatomical landmarks ensures comparable myocardial ischaemic area in all experiments. The suture was passed through a snare, the tightening and release of which induced ischaemia and reperfusion, respectively.

2.5. Infarct Size. Infarct size was measured in a separate series of experiments after 40 and 20 minutes of regional ischaemia, followed by 30 minutes of reperfusion, in wild-type ( $n=$ $10)$ and in ETB-deficient rats $(n=11)$. Infarct size was expressed as a percentage of ischaemic myocardial area at risk, as previously published from our laboratory $[33,37]$. The heart was perfused via the aorta with normal saline for 2 minutes, until all residual Krebs-Henseleit solution had been removed from the coronary arteries; subsequently, the coronary ligature was retightened at the same site, and $5 \mathrm{ml}$ green-fluorescent microspheres, $2-9 \mu \mathrm{m}$ in diameter (Duke Scientific Corp., Palo Alto, CA, USA), were infused over 5minutes. The right ventricle was removed, and the hearts were frozen at $-20^{\circ} \mathrm{C}$ for 24 hours and were sliced into $2 \mathrm{~mm}$-sections from apex to base. Following incubation in $1 \%$ triphenyltetrazolium chloride in isotonic phosphatebuffer solution (at $37^{\circ} \mathrm{C}, \mathrm{pH} 7.4$ ) for 20 minutes, the slices were immersed in 10\% formaldehyde solution for 24 hours. After placement between glass plates, the risk zone, the infarcted area, and the normal myocardium were identified under ultraviolet light $(\lambda=366 \mathrm{~nm})$ and traced on an acetate sheet. These areas were measured with the use of Image Tool (The University of Texas Health Science Center, San Antonio, TX USA); the volumes of infarct area and area at risk were expressed in $\mathrm{cm}^{3}$, and the percent ratio of infarct area/area at risk was calculated.

2.6. Statistical Analysis. All values are reported as mean \pm standard deviation. The infarct size values displayed a normal distribution (as per Kolmogorov-Smirnov test for normality) and were compared with the use of Student's $t$-test. Nonparametric analyses were performed to evaluate differences in haemodynamic variables, since normal distribution was not consistently present. Specifically, changes of haemodynamic variables over time were assessed with Friedman analysis of variance, whereas differences between groups in haemodynamic variables during reperfusion (expressed as percent changes compared to baseline) were examined with the use of Mann-Whitney $U$ test. Statistical trend was defined as $0.05<P<0.1$ and statistical significance as $P<0.05$.

\section{Results}

Changes in DP and EDP are graphically depicted in Figure 1 and in the first derivative of $\mathrm{LV}$ pressure (maximum $+d p / d t$ and maximum $-d p / d t$ ) in Figure 2.

3.1. 40-Minute Global Ischaemia. DP at the onset of reperfusion was lower $(P=0.035)$ in ETB-deficient rats, but subsequent values were comparable between the two groups. No significant differences were found in EDP between groups. Maximum $+d p / d t$ and maximum $-d p / d t$ were lower at the onset of reperfusion in ETB-deficient rats, but subsequent values were comparable between groups.

3.2. 20-Minute Global Ischaemia. DP at the onset of reperfusion was lower $(P=0.046)$ in ETB-deficient rats, but subsequent values were comparable in the two groups, as were all remaining haemodynamic variables. 
Global
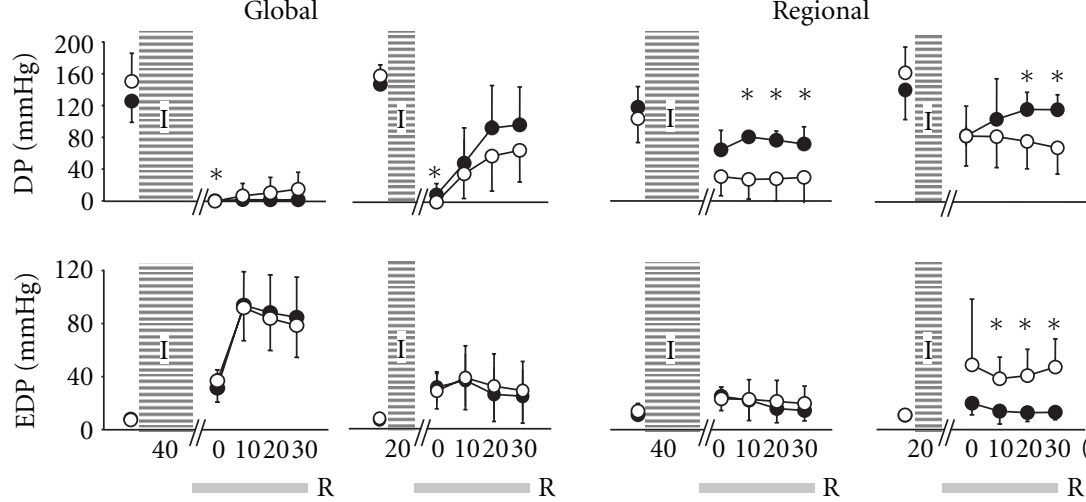

- Wild type

ETB deficient

FIGURE 1: Left-ventricular-developed pressure (LVDP, upper) and end-diastolic pressure (LVEDP, lower) after ischaemia (I)/reperfusion (R) in wild-type (dark dots) and ETB-deficient rats (white dots). Asterisk denotes significant differences between the two groups.
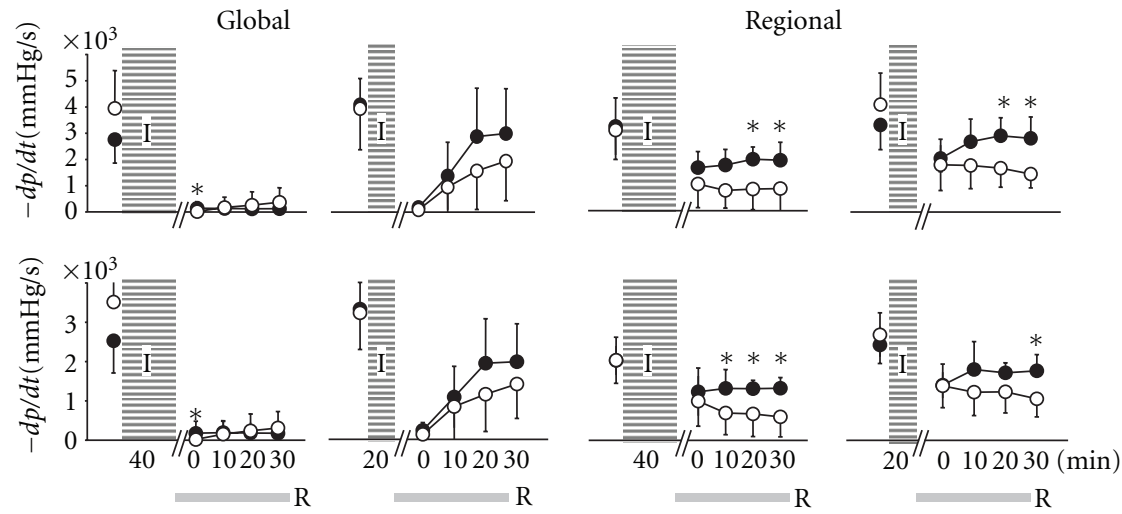

Wild type

FIGURE 2: Maximum values (maximum $+d p / d t$, upper) and minimum values (maximum $-d p / d t$, lower) of the first derivative of left ventricular pressure after ischaemia (I)/reperfusion (R) in wild-type (dark dots) and ETB-deficient rats (white dots). Asterisk denotes significant differences between the two groups.

3.3. 40-Minute Regional Ischaemia. There was a trend $(P=$ 0.086) towards lower DP values in ETB-deficient rats at the onset of reperfusion and this difference became significant (all $P<0.03$ ) at 10, 20, and 30 minutes after the onset of reperfusion. EDP did not differ between groups. Maximum $+d p / d t$ was lower (both $P<0.01$ ) in ETB-deficient rats at 20, and 30 minutes of reperfusion. A similar pattern was seen in maximum $-d p / d t$, with differences (all $P<0.03$ ) seen at 10 , 20 , and 30 minutes of reperfusion.

3.4. 20-Minute Regional Ischaemia. DP was lower (both $P<0.03)$ in ETB-deficient rats at 20 and 30 minutes of reperfusion. EDP was higher (all $P<0.02$ ) in ETB-deficient rats at 10,20 , and 30 minutes of reperfusion. Maximum $+d p / d t$ was marginally $(P=0.054)$ lower in ETB-deficient rats at 10 minutes and significantly (both $P<0.02$ ) lower at 20 and 30 minutes. Maximum $-d p / d t$ was significantly
$(P=0.0373)$ lower in ETB-deficient rats at the 30th minute of reperfusion.

3.5. Infarct Size after Regional Ischaemia-Reperfusion. As shown in Figure 3, 40 minutes of regional ischaemia, followed by 30 minutes of reperfusion, resulted in larger $(P=$ $0.00609)$ infarct size in ETB-deficient $(45.0 \pm 7.3 \%)$ than in wild-type $(31.5 \pm 4.4 \%)$ rats. A similar trend $(P=0.097)$ was seen after 20 minutes of regional ischaemia, followed by 30 minutes of reperfusion, with values at $24.5 \pm 7.5 \%$ in ETBdeficient and $16.3 \pm 7.9$ in wild-type animals.

3.6. Comparison between Regional and Global Ischaemia in Wild-Type Rats. Compared to the 40-minute regional ischaemia-reperfusion protocol, global ischaemia-reperfusion of equal duration in wild-type rats resulted in significantly (all $P<0.01$ ) lower $\mathrm{DP}$, maximum $+d p / d t$, 


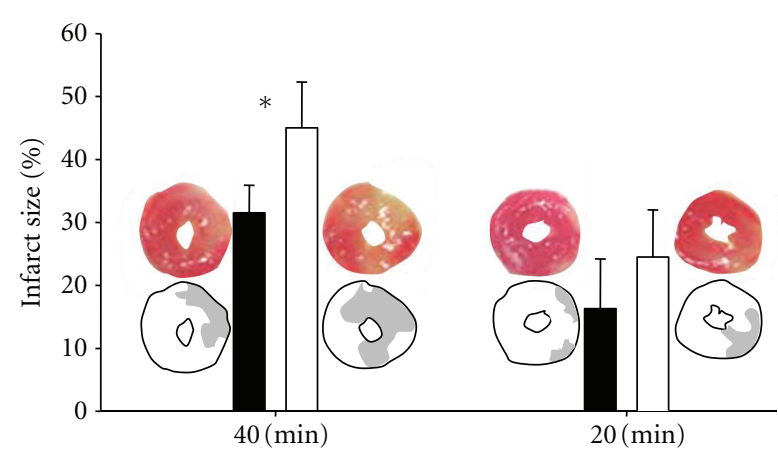

Wild type

$\square$ ETB deficient

Figure 3: Infarct size (expressed as percent ratio of necrotic area/area at risk) in wild-type (dark bars) and ETB-deficient rats (white bars) after 40- and 20-minute regional ischaemia. Asterisk denotes significant differences between the two groups. Representative examples of infarct size measurements (histological samples and schematic illustrations) are shown adjacent to the bars.

maximum $-d p / d t$, and higher EDP values. Similarly, 20 minutes of global ischaemia in wild-type rats resulted in significantly (all $P<0.01$ ) higher EDP values, compared to regional ischaemia of equal duration. In contrast, DP was lower $(P=0.0019)$ only at the onset of reperfusion after 20 minute global, compared to regional ischaemia. Maximum $+d p / d t$ and maximum $-d p / d t$ were lower only at the onset and at the 10th minute of reperfusion.

3.7. Comparison between Regional and Global Ischaemia in ETB-Deficient Rats. Compared to 40 minutes of regional ischaemia, global ischaemia of equal duration in ETBdeficient rats resulted in significantly (all $P<0.05$ ) lower maximum $+d p / d t$ (with the exception of values observed at the 30th minute), maximum $-d p / d t$, and higher EDP values. In contrast, recovery of DP was equally poor after either 40-minute global or regional ischaemia, with the exception of lower $(P=0.0011)$ values observed at the onset of reperfusion following global ischaemia. The recovery of all haemodynamic variables was comparable after 20 minutes of either global or regional ischaemia, except from values at the onset of reperfusion, when DP, maximum $+d p / d t$, and maximum $-d p / d t$ were lower $($ all $P<0.01$ ) after global ischaemia.

3.8. Comparison between 40- and 20-Minute Ischaemia in Wild-Type Rats. Compared to the 40-minute protocol, 20 minute global ischaemia-reperfusion was associated with improved recovery of LV function. This difference (both $P<0.01)$ was seen in DP at the 20th, and 30th minutes of reperfusion, in maximum $+d p / d t$ (all $P<0.05$ ) at the 10th, 20 th, and 30th minutes of reperfusion and in maximum $-d p / d t$ (both $P<0.02$ ) at the 20th and 30th minutes of reperfusion.
The same pattern of recovery of LV function was observed after 40- and 20-minute regional ischaemia protocols in DP (both $P<0.01$ ) at the 20th and 30th minute of reperfusion, in maximum $+d p / d t$ (all $P<0.05)$ at the 10 th, 20th, and 30th minute of reperfusion and in maximum $-d p / d t$ (both $P<0.02$ ) at the 20th and 30th minute of reperfusion.

3.9. Comparison between 40- and 20-Minute Ischaemia in ETB-Deficient Rats. Compared to the 40-minute protocol, 20-minute global ischaemia-reperfusion was associated with improved recovery of LV function. This difference was seen in DP, maximum $+d p / d t$, and maximum $-d p / d t$ during the entire reperfusion period (all $P<0.01$ ) and in EDP at the 10th, 20th, and 30th minutes of reperfusion (all $P<0.05$ ).

These differences were less pronounced in regional ischaemia protocols and were observed in DP [at the 10th minute $(P=0.032125)$, with a trend $(P=0.08647)$ present at the 20th minute of reperfusion]. No significant differences were observed in maximum $+d p / d t$ and in maximum- $d p / d t$. Surprisingly, EDP was higher after 20 than after 40 minutes of regional ischaemia; specifically, there was a trend $(P=$ $0.06329)$ towards higher values at the 10th minute, but significant differences were present at the 20th $(P=0.02227)$ and 30th $(P=0.00427)$ minutes of reperfusion.

\section{Discussion}

4.1. Main Findings. Despite ample research efforts, the pathophysiology of reperfusion injury after prolonged myocardial ischaemia remains incompletely understood. A substantial body of experimental data $[13,14]$ has implicated ET-1 as an important mediator in this process; ET-1 acts via ETA receptors, whereas the role of ETB receptors is unclear. Here, we report cytoprotective effects of ETB receptors on the ventricular myocardium after regional ischaemiareperfusion, evidenced by less myocardial necrosis and improved LV haemodynamic indices in wild-type, compared to ETB-deficient rats.

4.2. Comparison with Previous Studies. Previous studies investigating the role of ETA and ETB receptors after ischaemia-reperfusion produced diverse results, but these should be viewed with caution, given the limitations associated with pharmacologic blockade of ET receptors [1214]. In contrast, the use of the ETB-deficient rat model (developed and validated at Professor Yanagisawa's laboratory $[28,29])$ in our experiments permits more accurate assessment of the pathophysiologic role of ET receptors. Our findings confirm the conclusions on the role of ETA and ETB receptors derived by Yamamoto et al. [18], who used the same ETB-deficient rat strain. However, the main addition of the present work is the evaluation of two important parameters, namely, the type (regional versus global) and duration (40 versus 20 minutes) of ischaemia (all followed by a 30 -minute reperfusion period). 
4.3. Regional versus Global Ischaemia. Zero-flow global ischaemia protocols have been widely used in the ex vivo Langendorff-perfused rat-heart model. The main advantage is the comparable and easily reproducible extent of myocardial ischaemia, but the distant relevance with acute coronary artery occlusion in patients constitutes the main disadvantage of this model [20-22].

In the present study, we included protocols of ischaemia, induced by ex vivo left anterior descending coronary artery ligation. This technique, originally described in 1975 [38], combines the closer resemblance with myocardial ischaemia/infarction in man, with the accuracy of measurements offered by the ex vivo heart model; based on these advantages, this method is considered of value in the investigation of myocardial ischaemia/infarction $[20,21]$. Our findings of more physiological values after regional ischaemia, as opposed to the marked postischaemic LV dysfunction, observed mainly after 40-minute-global ischaemia, reinforce these considerations [20, 21]. Moreover, the less prominent LV dysfunction, produced by regional ischaemia, permitted the more accurate evaluation of the effects of ETB receptors on the ventricular myocardium.

In our experiments, postischaemic LV dysfunction after 40 minutes of global ischaemia was more pronounced (in wild-type and in ETB-deficient animals) compared to that reported by Yamamoto et al. [18]. Since heart rate correlates with cardiac work [19], lower heart rate may be cardioprotective $[19,34]$; therefore, the difference in postischaemic LV dysfunction between our experiments and those by Yamamoto et al. [18] could be attributed to the effects of higher heart rate in our experiments, produced by atrial pacing at a rate of 300 beats per minute. Indeed, ventricular pacing was shown to enhance postischaemic ventricular dysfunction in this setting [19], although ventricular asynchrony induced by such pacing constitutes a welldescribed detrimental factor [39].

4.4. 40-versus 20-Minute Ischaemia. We evaluated the duration of the ischaemic period, by comparing two commonly used protocols [20-22]. In agreement with previous studies [19], we report exaggerated postischaemic LV dysfunction with 40- more than with 20-minute ischaemia, this difference being more prominent after global than after regional ischaemia.

The correlation between ischaemia duration (in the ischaemia-reperfusion isolated rat-heart model) and the time to treatment onset (thrombolysis or primary percutaneous coronary intervention) in patients with myocardial infarction is unknown. A recent meta-analysis [40] indicated time intervals from symptom onset to intervention at the range of 90-140 minutes, and these figures appear further improved in more recent programmes [41, 42]. Based on this information, we propose that the 20 -minute regional ischaemia protocol applies most to clinical practice, whereas longer ischaemic periods are still relevant but correspond to progressively smaller proportion of patients.
4.5. Mechanisms of ETB Receptor-Induced Cytoprotection. The mechanisms of ventricular cytoprotective effects of ETB receptors after ischaemia-reperfusion remain under investigation. ETB receptors may be involved in the local sequestration and clearance of ET-1, not only under normal, but also during ischaemic conditions [43]. Increased ET1 locally in the myocardium in the chronic absence of ETB receptors may exert vasoconstrictive actions via ETA receptors, thereby increasing coronary artery resistance [43]. Thus, in our experiments, ETA receptor-mediated responses may have been exaggerated in ETB-deficient animals. These notions are reinforced by the experiments of Yamamoto et al. [18], who found exaggerated coronary-flow reduction in ETB-deficient rats, which was restored after selective ETA receptor blockade. In fact, our experiments lend further support to this view, as the differences between wild-type and ETB-deficient rats were relatively minor under conditions of constant coronary flow, in the setting of 40-minute global ischaemia. However, our results point out towards additional underlying mechanisms, based (a) on the different haemodynamic responses still being present after global or (mainly) regional ischaemia (followed by reperfusion) and (b) on the decreased infarct size in wild-type rats after regional ischaemia (followed by reperfusion).

Yamamoto et al. [18] reported enhanced norepinephrine overflow in ETB-deficient rats, suggesting an essential role of ETB receptors in the regulation of local myocardial sympathetic response under ischaemic conditions. This finding was confirmed in a previous study by our group [44], using the in vivo model of coronary artery ligation in the same ETB-deficient rat strain; in this study, we found markedly higher sympathetic activation in ETB-deficient rats, assessed by fast Fourier analysis of heart rate variability, as well as by measurement of serum epinephrine and norepinephrine levels [44]. The well-described [45] mechanisms of norepinephrine-induced acceleration of cell death in the ischaemic ventricular myocardium include augmented myocardial oxygen demand, mediated by increased heart rate and contractility, as well as decreased oxygen supply secondary to coronary artery vasoconstriction.

4.6. ETB Receptor-Induced Cytoprotection and IschaemiaReperfusion Protocols. In our experiments, the differences in haemodynamic variables between wild-type and ETBdeficient rats were less evident after global ischaemia, providing another potential explanation for the discrepant conclusions of previous studies $[15,18]$ reported in this setting. In contrast, the recovery of left ventricular function was poorer in ETB-deficient rats after regional ischaemia, indicating a pathophysiologic role of ETB receptors in more clinically applicable experimental conditions.

A paradoxical finding in our experiments was the higher EDP observed in ETB- deficient rats after 20 than after 40 minutes of regional ischaemia. Although difficult to explain, this is in line with our previous observations [44], indicating a prominent role of ETB receptors in sympathetic activation only during the early phase of infarction, with strikingly diminished effects during subsequent stages. 
The time course of norepinephrine release from the sympathetic nerve terminals in the ventricular myocardium in response to prolonged ischaemia has been subject of intense research [46]. During the early phase, this process occurs initially by norepinephrine exocytosis but is subsequently greatly enhanced by reverse-mode function of the norepinephrine transporter, thereby releasing the accumulated free axoplasmic norepinephrine. ET- 1 has been shown to promote nonexocytotic norepinephrine release early after prolonged ischaemia [47]; this action occurs via ETA receptor stimulation, resulting in axoplasmic sodium accumulation and reversal of norepinephrine transporter $[18,47]$. ETB receptors appear to counteract this effect, evidenced by marked elevation of norepinephrine release after selective ETB receptor blockade in isolated ischaemic guinea pig hearts [48]. However, during subsequent stages, this protective effect of ETB receptors diminishes, possibly due to axoplasmic norepinephrine depletion [44].

4.7. Strengths and Limitations of the Study. We examined the role of ETB receptors using four ischaemia-reperfusion protocols in the isolated Langendorff-perfused rat heart. The evaluation of ischaemia duration and the induction of clinically relevant ischaemia represent major strengths of our work. However, three limitations should be acknowledged. First, coronary flow was held constant throughout the experiments, enabling the assessment of postischaemic LV dysfunction without the confounding factor of coronary autoregulation; nonetheless, the addition of protocols using constant perfusion pressure would have permitted the assessment of the effects of ETB receptors on coronary vascular resistance. Second, our study did not address the issue of the pathophysiologic mediators of ETB receptor activation. More specifically, we did not include measurements of norepinephrine levels in the perfusate, that could have expanded previous findings [18, 44]. Third, our protocol did not include the calculation of infarct size after global ischaemia-reperfusion; although measurement of diffuse necrosis is more difficult, its addition in the protocol could have increased the value of our results.

\section{Conclusions}

ETB receptors exert cytoprotective effects on the ventricular myocardium after ischaemia-reperfusion in the isolated, Langendorff-perfused working rat heart. These effects are more prominent after regional than after global ischaemia (followed by reperfusion). Longer periods of ischaemia suppress the recovery of LV function after reperfusion, but the role of ETB receptors may be more important during the early phases.

\section{Funding}

This research was supported by a grant from the Cardiovascular Research Institute, Ioannina and Athens, Greece.

\section{Disclosure}

The authors had full access to the data and take responsibility for the integrity of the data. All the authors have read and agreed to the paper as written. None of the authors has a conflict of interests of any kind.

\section{Acknowledgments}

Eleftheria Karampela, RN, Kalliopi Tsarea, Maria Karamperi, and Argyro Zacharioudaki, DVM, assisted during the experiments. Eleni V. Goga, MS, coordinated this research.

\section{References}

[1] P. A. Heidenreich, J. G. Trogdon, O. A. Khavjou et al., "Forecasting the future of cardiovascular disease in the United States: a policy statement from the American Heart Association," Circulation, vol. 123, no. 8, pp. 933-944, 2011.

[2] H. K. Eltzschig and T. Eckle, "Ischemia and reperfusion-from mechanism to translation," Nature Medicine, vol. 17 , no. 11, pp. 1391-1401, 2011.

[3] M. Yanagisawa, H. Kurihara, S. Kimura et al., "A novel potent vasoconstrictor peptide produced by vascular endothelial cells," Nature, vol. 332, no. 6163, pp. 411-415, 1988.

[4] M. Barton and M. Yanagisawa, "Endothelin: 20 Years from discovery to therapy," Canadian Journal of Physiology and Pharmacology, vol. 86, no. 8, pp. 485-498, 2008.

[5] P. Molenaar, G. O'Reilly, A. Sharkey et al., "Characterization and localization of endothelin receptor subtypes in the human atrioventricular conducting system and myocardium," Circulation Research, vol. 72, no. 3, pp. 526-538, 1993.

[6] S. Tsuji, A. Sawamura, H. Watanabe, K. Takihara, S. E. Park, and J. Azuma, "Plasma endothelin levels during myocardial ischemia and reperfusion," Life Sciences, vol. 48, no. 18, pp. 1745-1749, 1991.

[7] T. Watanabe, N. Suzuki, N. Shimamoto, M. Fujino, and A. Imada, "Endothelin in myocardial infarction," Nature, vol. 344, no. 6262, p. 114, 1990.

[8] T. Watanabe, N. Suzuki, N. Shimamoto, M. Fujino, and A. Imada, "Contribution of endogenous endothelin to the extension of myocardial infarct size in rats," Circulation Research, vol. 69, no. 2, pp. 370-377, 1991.

[9] J. P. Loennechen, A. Støylen, V. Beisvag, U. Wisløff, and Ø. Ellingsen, "Regional expression of endothelin-1, ANP, IGF-1, and LV wall stress in the infarcted rat heart," American Journal of Physiology, vol. 280, no. 6, pp. H2902-H2910, 2001.

[10] A. Proven, H. L. Roderick, S. J. Conway et al., "Inositol 1,4,5-trisphosphate supports the arrhythmogenic action of endothelin-1 on ventricular cardiac myocytes," Journal of Cell Science, vol. 119, no. 16, pp. 3363-3375, 2006.

[11] C. Steenbergen, E. Murphy, J. A. Watts, and R. E. London, "Correlation between cytosolic free calcium, contracture, ATP, and irreversible ischemic injury in perfused rat heart," Circulation Research, vol. 66, no. 1, pp. 135-146, 1990.

[12] J. Pernow and Q. D. Wang, "Endothelin in myocardial ischaemia and reperfusion," Cardiovascular Research, vol. 33, no. 3, pp. 518-526, 1997.

[13] P. Cernacek, D. J. Stewart, J. C. Monge, and J. L. Rouleau, "The endothelin system and its role in acute myocardial infarction," Canadian Journal of Physiology and Pharmacology, vol. 81, no. 6, pp. 598-606, 2003. 
[14] C. L. Wainwright, C. McCabe, and K. A. Kane, "Endothelin and the ischaemic heart," Current Vascular Pharmacology, vol. 3, no. 4, pp. 333-341, 2005.

[15] P. H. Dagassan, V. Brue, M. Clozel, and J. P. Clozel, "Role of endothelin during reperfusion after ischemia in isolated perfused rat heart," Journal of Cardiovascular Pharmacology, vol. 24, no. 6, pp. 867-874, 1994.

[16] J. Y. Lee, R. B. Warner, A. L. Adler, and T. J. Opgenorth, "Endothelin ET(A) receptor antagonist reduces myocardial infarction induced by coronary artery occlusion and reperfusion in the rat," Pharmacology, vol. 49, no. 5, pp. 319-324, 1994.

[17] N. F. Kelland, R. E. Kuc, D. L. McLean et al., "Endothelial cell-specific ETB receptor knockout: autoradiographic and histological characterisation and crucial role in the clearance of endothelin-1," Canadian Journal of Physiology and Pharmacology, vol. 88, no. 6, pp. 644-651, 2010.

[18] S. Yamamoto, N. Matsumoto, M. Kanazawa et al., "Different contributions of endothelin-A and endothelin-B receptors in postischemic cardiac dysfunction and norepinephrine overflow in rat hearts," Circulation, vol. 111, no. 3, pp. 302309, 2005.

[19] J. P. Headrick, J. Peart, B. Hack, A. Flood, and G. Paul Matherne, "Functional properties and responses to ischaemiareperfusion in Langerdorff perfused mouse heart," Experimental Physiology, vol. 86, no. 6, pp. 703-716, 2001.

[20] K. Ytrehus, "The ischemic heart-experimental models," Pharmacological Research, vol. 42, no. 3, pp. 193-203, 2000.

[21] M. Skrzypiec-Spring, B. Grotthus, A. Szelag, and R. Schulz, "Isolated heart perfusion according to Langendorff-Still viable in the new millennium," Journal of Pharmacological and Toxicological Methods, vol. 55, no. 2, pp. 113-126, 2007.

[22] P. D. Verdouw, M. A. Van Den Doel, S. De Zeeuw, and D. J. Duncker, "Animal models in the study of myocardial ischaemia and ischaemic syndromes," Cardiovascular Research, vol. 39, no. 1, pp. 121-135, 1998.

[23] D. H. Fitchett, P. Theroux, J. M. Brophy et al., "Assessment and management of acute coronary syndromes (ACS): a Canadian perspective on current guideline-recommended treatmentpart 2: ST-segment elevation myocardial infarction," Canadian Journal of Cardiology, vol. 27, no. 6, supplement, pp. S402-S412, 2011.

[24] F. Brunner, E. F. Du Toit, and L. H. Opie, "Endothelin release during ischaemia and reperfusion of isolated perfused rat hearts," Journal of Molecular and Cellular Cardiology, vol. 24, no. 11, pp. 1291-1305, 1992.

[25] G. G. Baltogiannis, D. G. Tsalikakis, A. C. Mitsi et al., "Endothelin receptor-a blockade decreases ventricular arrhythmias after myocardial infarction in rats," Cardiovascular Research, vol. 67, no. 4, pp. 647-654, 2005.

[26] T. M. Kolettis, G. G. Baltogiannis, D. G. Tsalikakis et al., "Effects of dual endothelin receptor blockade on sympathetic activation and arrhythmogenesis during acute myocardial infarction in rats," European Journal of Pharmacology, vol. 580, no. 1-2, pp. 241-249, 2008.

[27] T. M. Kolettis, K. Kontaras, I. Spartinos et al., "Dosedependent effects of sildenafil on post-ischaemic left ventricular function in the rat isolated heart," Journal of Pharmacy and Pharmacology, vol. 62, no. 3, pp. 346-351, 2010.

[28] C. E. Gariepy, S. C. Williams, J. A. Richardson, R. E. Hammer, and M. Yanagisawa, "Transgenic expression of the endothelin$\mathrm{B}$ receptor prevents congenital intestinal aganglionosis in a rat model of Hirschsprung disease," Journal of Clinical Investigation, vol. 102, no. 6, pp. 1092-1101, 1998.
[29] C. E. Gariepy, T. Ohuchi, S. C. Williams, J. A. Richardson, and M. Yanagisawa, "Salt-sensitive hypertension in endothelin-B receptor-deficient rats," Journal of Clinical Investigation, vol. 105, no. 7, pp. 925-933, 2000.

[30] C. E. Gariepy, D. T. Cass, and M. Yanagisawa, "Null mutation of endothelin receptor type $\mathrm{B}$ gene in spotting lethal rats causes aganglionic megacolon and white coat color," Proceedings of the National Academy of Sciences of the United States of America, vol. 93, no. 2, pp. 867-872, 1996.

[31] M. G. Perry, M. M. Molero, A. D. Giulumian et al., "EtB receptor-deficient rats exhibit reduced contraction to ET-1 despite an increase in ETA receptors," American Journal of Physiology, vol. 281, no. 6, pp. H2680-H2686, 2001.

[32] D. D. Ivy, I. F. Mcmurtry, M. Yanagisawa et al., "Endothelin B receptor deficiency potentiates ET-1 and hypoxic pulmonary vasoconstriction," American Journal of Physiology, vol. 280, no. 5, pp. L1040-L1048, 2001.

[33] V. C. Varnavas, K. Kontaras, C. Glava et al., "Chronic skeletal muscle ischemia preserves coronary flow in the ischemic rat heart," American Journal of Physiology, vol. 301, no. 4, pp. H1229-H1235, 2011.

[34] A. Tosaki, S. Balint, and L. Szekeres, "Pacing reperfusion induced arrhythmias: protection by slow heart rate in the rat heart," Cardiovascular Research, vol. 22, no. 11, pp. 818-825, 1988.

[35] D. A. Elaiopoulos, D. G. Tsalikakis, M. G. Agelaki et al., "Growth hormone decreases phase II ventricular tachyarrhythmias during acute myocardial infarction in rats," Clinical Science, vol. 112, no. 7-8, pp. 385-391, 2007.

[36] M. G. Agelaki, C. Pantos, P. Korantzopoulos et al., "Comparative antiarrhythmic efficacy of amiodarone and dronedarone during acute myocardial infarction in rats," European Journal of Pharmacology, vol. 564, no. 1-3, pp. 150-157, 2007.

[37] Z. S. Kyriakides, E. K. Iliodromitis, C. Papadopoulos, N. Sourlas, and D. T. Kremastinos, "Atrioventricular pacing does not increase infarct size in the in situ rabbit heart," Canadian Journal of Cardiology, vol. 13, no. 6, pp. 603-606, 1997.

[38] G. J. Kannengiesser, W. F. Lubbe, and L. H. Opie, "Experimental myocardial infarction with left ventricular failure in the isolated perfused rat heart. Effects of isoproterenol and pacing," Journal of Molecular and Cellular Cardiology, vol. 7, no. 2, pp. 135-151, 1975.

[39] Z. S. Kyriakides, A. G. Manolis, and T. M. Kolettis, "The effects of ventricular asynchrony on myocardial perfusion," International Journal of Cardiology, vol. 119, no. 1, pp. 3-9, 2007.

[40] S. C. Brooks, K. S. Allan, M. Welsford, P. Richard Verbeek, H. R. Arntz, and L. J. Morrison, "Prehospital triage and direct transport of patients with ST-elevation myocardial infarction to primary percutaneous coronary intervention centres: a systematic review and meta-analysis," Canadian Journal of Emergency Medicine, vol. 11, no. 5, pp. 481-492, 2009.

[41] M. R. Le May, D. Y. So, R. Dionne et al., "A citywide protocol for primary PCI in ST-segment elevation myocardial infarction," New England Journal of Medicine, vol. 358, no. 3, pp. 231-240, 2008.

[42] J. S. De Villiers, T. Anderson, J. D. McMeekin, R. C. M. Leung, and M. Traboulsi, "Expedited transfer for primary percutaneous coronary intervention: a program evaluation," Canadian Medical Association Journal, vol. 176, no. 13, pp. 1833-1838, 2007.

[43] F. Brunner and A. M. Doherty, "Role of ET(B) receptors in local clearance of endothelin-1 in rat heart: studies with the 
antagonists PD 155080 and BQ-788," FEBS Letters, vol. 396, no. 2-3, pp. 238-242, 1996.

[44] D. L. Oikonomidis, D. G. Tsalikakis, G. G. Baltogiannis et al., "Endothelin-B receptors and ventricular arrhythmogenesis in the rat model of acute myocardial infarction," Basic Research in Cardiology, vol. 105, no. 2, pp. 235-245, 2010.

[45] G. Rona, "Catecholamine cardiotoxicity," Journal of Molecular and Cellular Cardiology, vol. 17, no. 4, pp. 291-306, 1985.

[46] A. Schomig, M. Haass, and G. Richardt, "Catecholamine release and arrhythmias in acute myocardial ischaemia," European Heart Journal, vol. 12, pp. 38-47, 1991.

[47] L. Fliegel, "Regulation of myocardial $\mathrm{Na}^{+} / \mathrm{H}^{+}$exchanger activity," Basic Research in Cardiology, vol. 96, no. 4, pp. 301305, 2001.

[48] M. Isaka, A. Kudo, M. Imamura, H. Kawakami, and K. Yasuda, "Endothelin receptors, localized in sympathetic nerve terminals of the heart, modulate norepinephrine release and reperfusion arrhythmias," Basic Research in Cardiology, vol. 102, no. 2, pp. 154-162, 2007. 


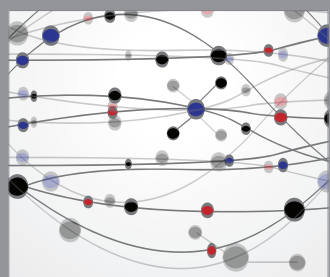

The Scientific World Journal
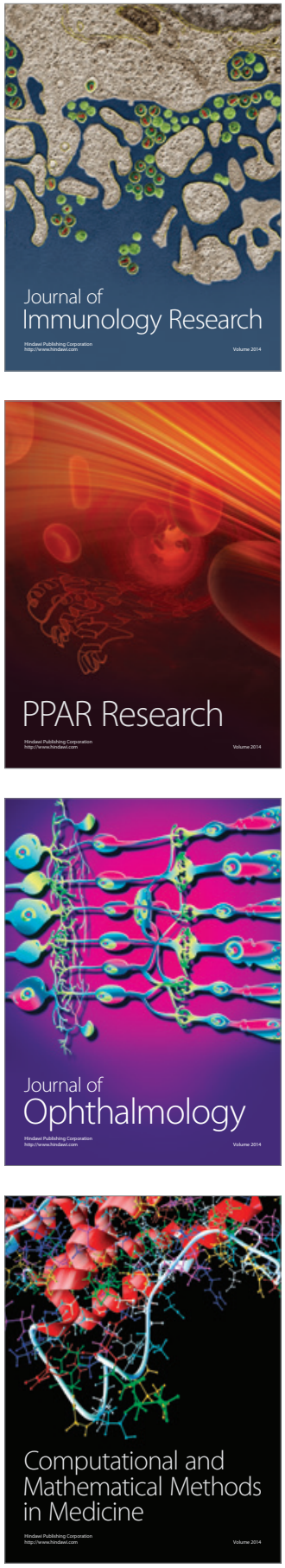

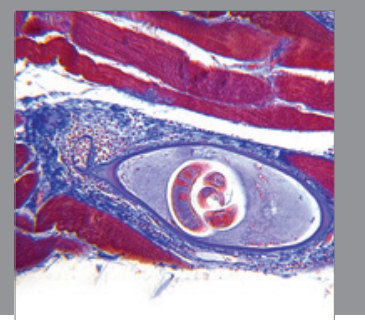

Gastroenterology

Research and Practice
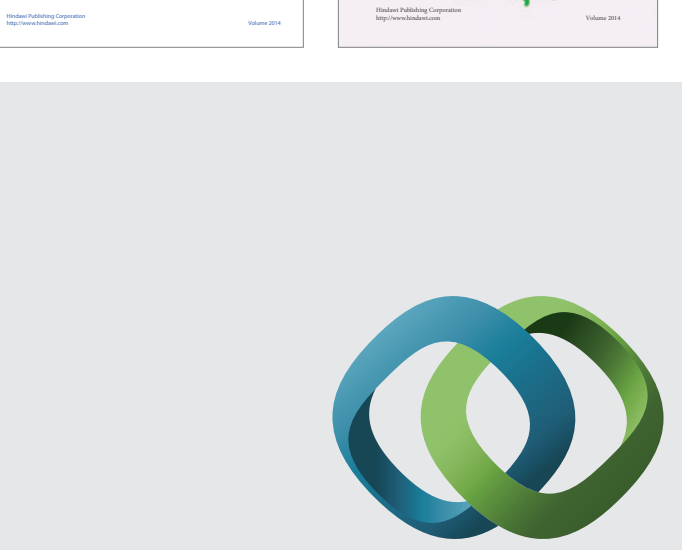

\section{Hindawi}

Submit your manuscripts at

http://www.hindawi.com
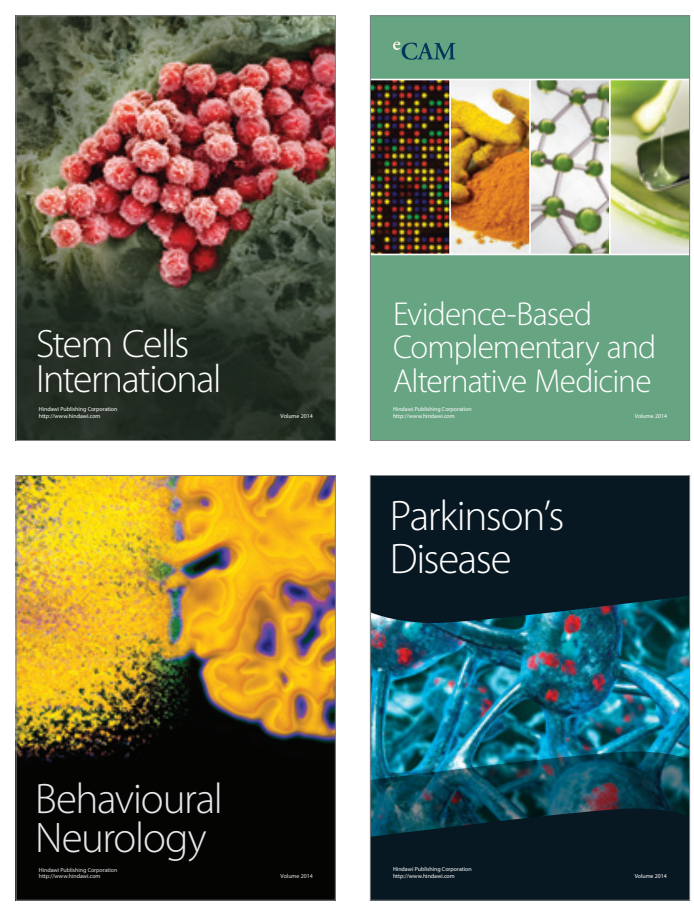

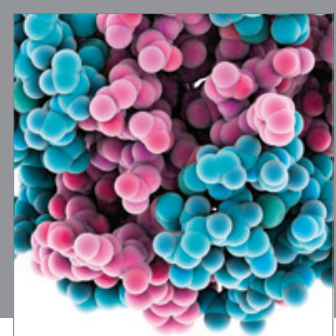

Journal of
Diabetes Research

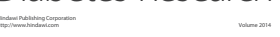

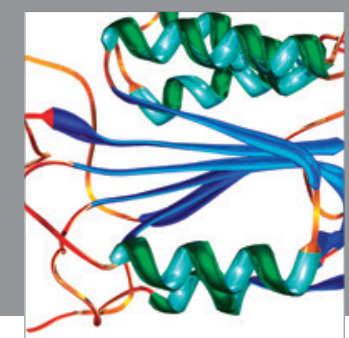

Disease Markers
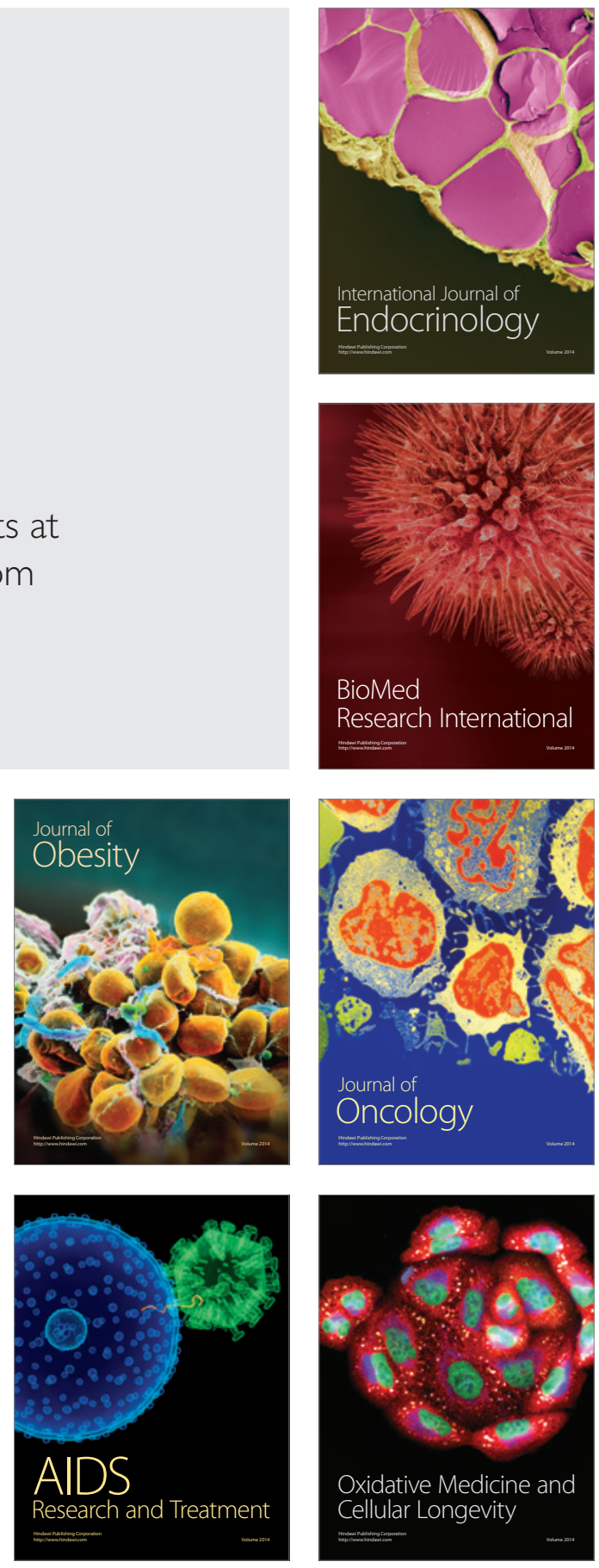